\title{
Erratum to: Architecture for development of adaptive on-line prediction models
}

\author{
Petr Kadlec · Bogdan Gabrys
}

Published online: 1 February 2013

(C) Springer-Verlag Berlin Heidelberg 2013

\section{Erratum to: Memetic Comp. (2009) 1:241-269 DOI 10.1007/s12293-009-0017-8}

Due to an unfortunate turn of events this article has been published with errors in some of the equations ${ }^{1}$ for which the authors would like to apologise.

The authors would also like to note that the correct formulas given below have been used in all the experiments reported in the paper, hence the experimental results remain valid and are not affected by this erratum.

1. As the two-dimensional probability density functions (PDFs) have been used together with the Parzen window method of their estimation for the construction of the LEC descriptors, Eq. (14) should read:

$$
L_{(m), j}=\sum_{i \in \mathcal{D}^{v a l}} w_{(m), i} \Phi\left(\mu_{i}, \Sigma\right) \quad \text { s.t. } \sum_{i \in \mathcal{D}^{v a l}} w_{(m), i}=1
$$

\footnotetext{
1 The authors would like to thank Dr Marcin Budka for bringing the errors to their attention and providing the corrected formulas. This has been carried out in the follow up INFER project where all the formulas and algorithms presented in the paper have been independently checked and verified.
}

The online version of the original article can be found under doi:10.1007/s12293-009-0017-8.

\section{P. Kadlec $(\bowtie) \cdot$ B. Gabrys}

Smart Technology Research Centre,

Bournemouth University, BH12 5BB, Fern Barrow, Poole, UK

e-mail: petr.kadlec@bournemouth.ac.uk

B. Gabrys

e-mail: BGabrys@bournemouth.ac.uk which emphasises that the weights $w_{(m), i}$ must add up to 1 and preserves the interpretations of $L_{(m), j}$ as a PDF.

2. In order for Eq. (19) to map the error $e_{(m)}$ into a performance index $u_{(m)}$, so that the best performing local expert receives a weight (score) equal to 1 and the weights of the remaining local experts decay exponentially with the increasing error, whereas the median error is mapped to the value 0.5, Eq. (19) should take the following form:

$u_{(m)}=\exp \left(\frac{e_{(m)} \log (0.5)}{\operatorname{med}(\mathbf{e})}\right)$

where 'log' denotes the natural logarithm.

3 . The a priori probability $p(j)$ for the $j$-th expert is missing in the denominator of the original Eq. (24) and the indexes in the denominator have been changed from $m$ to $j$. Equation (24) and the paragraph immediately below it should therefore have the following form:

$$
\begin{aligned}
v_{(m)}\left(\mathbf{x}, f_{(m)}^{L E}\right) & =p\left(m \mid \mathbf{x}, f_{(m)}^{L E}\right) \\
& =\frac{p\left(\mathbf{x}, f_{(m)}^{L E} \mid m\right) p(m)}{\sum_{j=1}^{N_{e n s}} p\left(\mathbf{x}, f_{(j)}^{L E} \mid j\right) p(j)}
\end{aligned}
$$

where $p(m)$ is the a priori probability of the $m$-th Local Expert (in our implementation equal for all LEs but in general it can be used to prioritise between them), $\sum_{j=1}^{N_{\text {ens }}} p\left(\mathbf{x}, f_{(j)}^{L E} \mid j\right) p(j)$ is a normalisation factor and $p\left(\mathbf{x}, f_{(m)}^{L E} \mid m\right)$ the likelihood of $\boldsymbol{x}$ and the local expert which can be calculated by reading the descriptor of the $m$-th Local expert $\mathcal{L}_{(m)}$ : 